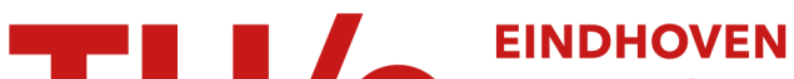 \\ UNIVERSITY OF \\ TECHNOLOGY
}

\section{Optimizing airspace closure with respect to politicians' egos}

Citation for published version (APA):

Kostitsyna, l., Löffler, M., \& Polishchuk, V. (2014). Optimizing airspace closure with respect to politicians' egos. In A. Ferro, F. Luccio, \& P. Widmayer (Eds.), Proc. 7th International Conference on Fun with Algorithms (FUN) (pp. 264-276). (Lecture Notes in Computer Science; Vol. 8496). Springer. https://doi.org/10.1007/978-3-31907890-8_23

DOI:

10.1007/978-3-319-07890-8_23

Document status and date:

Published: 01/01/2014

\section{Document Version:}

Accepted manuscript including changes made at the peer-review stage

\section{Please check the document version of this publication:}

- A submitted manuscript is the version of the article upon submission and before peer-review. There can be important differences between the submitted version and the official published version of record. People interested in the research are advised to contact the author for the final version of the publication, or visit the $\mathrm{DOI}$ to the publisher's website.

- The final author version and the galley proof are versions of the publication after peer review.

- The final published version features the final layout of the paper including the volume, issue and page numbers.

Link to publication

\section{General rights}

Copyright and moral rights for the publications made accessible in the public portal are retained by the authors and/or other copyright owners and it is a condition of accessing publications that users recognise and abide by the legal requirements associated with these rights.

- Users may download and print one copy of any publication from the public portal for the purpose of private study or research.

- You may not further distribute the material or use it for any profit-making activity or commercial gain

- You may freely distribute the URL identifying the publication in the public portal.

If the publication is distributed under the terms of Article 25fa of the Dutch Copyright Act, indicated by the "Taverne" license above, please follow below link for the End User Agreement:

www.tue.nl/taverne

Take down policy

If you believe that this document breaches copyright please contact us at:

openaccess@tue.nl

providing details and we will investigate your claim. 


\title{
Optimizing Airspace Closure with Respect to Politicians' Egos
}

\author{
Irina Kostitsyna ${ }^{1}$, Maarten Löffler ${ }^{2}$, and Valentin Polishchuk ${ }^{3}$ \\ 1 Department of Mathematics and Computer Science, Eindhoven University of Technology, \\ The Netherlands. i. kostitsyna@tue.nl \\ 2 Department of Information and Computing Sciences, Utrecht University, The Netherlands. \\ m. lofflerduu.nl \\ 3 Communications and Transport Systems, ITN, Linköping University, Sweden. \\ valentin.polishchukeliu.se
}

\begin{abstract}
When a president is landing at a busy airport, the airspace around the airport closes for commercial traffic. We show how to schedule the presidential squadron so as to minimize its impact on scheduled civilian flights; to obtain an efficient solution we use a "rainbow" algorithm recoloring aircraft on the fly as they are stored in a special type of forest. We also give a data structure to answer the following query efficiently: Given the president's ego (the requested duration of airspace closure), when would be the optimal time to close the airspace? Finally, we study the dual problem: Given the time when the airspace closure must start, what is the longest ego that can be tolerated without sacrificing the general traffic? We solve the problem by drawing a Christmas tree in a delay diagram; the tree allows one to solve also the query version of the problem.
\end{abstract}

\section{Introduction}

Airspace closure due to military activities is a pain for civilian air traffic controllers (ATCOs), pilots, airlines and other stakeholders; the issue is especially notorious in countries with heavy military control of the skies (such as China). Military flight operations range from strike and defense missions to drills to humanitarian airdrops. The missions are impossible to reschedule, and military ATCOs are entitled to ceasing airspace from civilian use at any time when the traffic could conflict with the mission aircraft. Drills are better in this regard because they are planned in advance, but nevertheless airspace closure due to drills harms commercial airlines. On the contrary, humanitarian aid delivery typically has little effect on general air traffic - not the least due to the fact that the aid is often delivered to places far from mainstream airports.

There is one activity involving military air force, however, whose scheduling most certainly could have been done wiser than it is done today: air transfer of VIPs (presidents and other high-ranked politicians). We trust that planners of such activities are instructed by their superiors (the VIPs at hand) to take civilian needs into account when planning the flights - all VIPs are conscientious citizens putting needs of the people above their personal comfort. Unfortunately, no matter how hard the planners strive to follow the instructions, the civilians do get annoyed with delays caused by VIP flights. For instance, the recent visit of the US President to Sweden disrupted air traffic to Stockholm area and gave rise to heated discussions among professionals in the Malmö 
Air Traffic Control Center (in the south of Sweden) about possible measures that could have been taken to diminish the disruption [2]. Apparently, the only reason preventing the planners of VIP flights from requesting airspace closure with minimum impact on scheduled traffic is the absence of efficient algorithms for computing the optimum.

In this paper we set out to alleviate the difficulty by providing algorithms for deciding the optimal airspace closure timing. Employing our solutions will make the general public happier about VIPs, which will eventually pay back to politicians at future elections.

\subsection{Model}

For every aircraft the optimal flight plan exists (it can be fuel-optimal or time-optimal or optimal according to another objective) which includes both the altitude profile and the speed at every point along the path. Whether the aircraft is able to execute such a plan depends heavily on the other aircraft around. In the uncongested enroute portion of the flight aircraft with similar headings can generally "overtake" each other; to quote Director General of Luftfartsverket (Swedish air navigation service provider) [1], the uncontrolled oceanic airspace witnesses "air race over the Atlantic" on a daily basis. On the contrary, in the vicinity of an airport, the arrival manager sequences aircraft "ducks-in-a-row" to the approach (the final phase of the flight); here, faster aircraft (those whose desired speed is larger) must slow down in order to maintain separation from the preceding slower plane. This latter scenario is the one considered in this paper.

Assume that the approach to an airport is a "single-lane road" of length 1 . The approach does not have to be a straight-line segment (in fact, in real world, approaches to many airports are curved); the important thing is that it is a one-dimensional curve. Aircraft enter the approach at times $t_{1}, \ldots, t_{n} \in[0,1]$ (known from the schedules or flight plans) and have desired speeds $v_{1}, \ldots, v_{n}$ (known from communication between pilots and ATCOs or from automated flight management systems); $n$ is the number of the aircraft. For simplicity assume all $t_{i} \mathrm{~s}$ and all $v_{i} \mathrm{~s}$ are distinct. In absence of the other planes, aircraft $i$ would traverse the approach uniformly at speed $v_{i}$, arriving at the airport at time $\tau_{i}=t_{i}+1 / v_{i}$; this is an oversimplification, but our solutions can be modified to work with arbitrary desired speed profiles. Since passing is not allowed on the approach, any aircraft must slow down to the speed of the preceding plane as soon as the aircraft catches up the plane. In other words, we assume that aircraft have 0 length and can follow each other without gaps; this is also an oversimplification, but again, our algorithms can work under the requirement of minimum safe separation distances between aircraft as well (in reality, the minimum miles-in-trail restriction is not uniform - it depends on the types of aircraft, with light aircraft having to stay farther behind a heavier one to avoid wake vortices). That is, in the $t x$-plane, the desired trajectory of aircraft $i$ is the segment $s_{i}$ going from $\left(t_{i}, 0\right)$ to $\left(\tau_{i}, 1\right)$; we identify $i$ with the segment and use $i$ or $s_{i}$ interchangeably. However, if the aircraft catches up with a slower plane they start moving together at the slower speed; thus the actual aircraft location on the approach is a concave piecewise-linear function of time, and in the $t x$ plane the trajectories get merged into trees corresponding to platoons of aircraft (Fig. 1, left). 

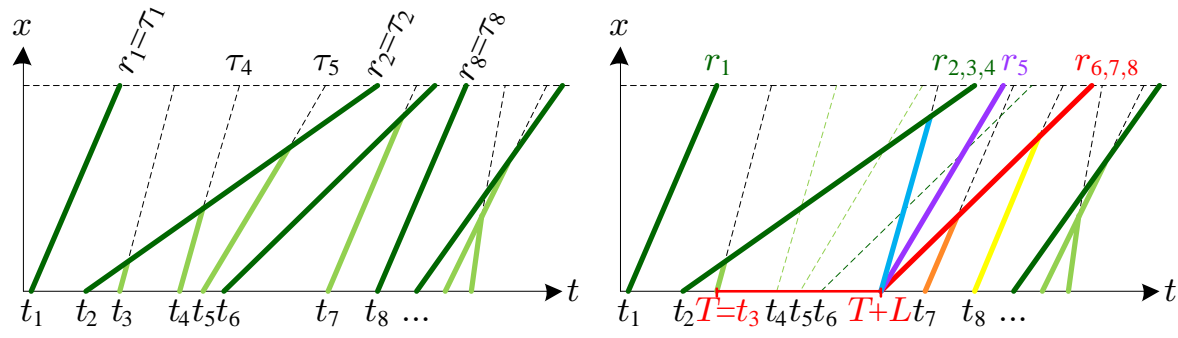

Fig. 1: Left: The desired trajectories are dashed, the actual trajectories are green; the green segment starting at $\left(t_{i}, 0\right)$ is the part that aircraft $i$ travels with its desired speed $v_{i}$. Trees correspond to platoons of aircraft landing at the same time; dark green are platoon heads. Right: The trajectories change due to the closure (the aircraft are colored according to our algorithm in Section 2)

Let $r_{i}$ denote the actual time when aircraft $i$ lands $\left(r_{i}=\tau_{i}\right.$ for platoon heads); the points $\left(r_{i}, 1\right)$ are roots of platoon trees. The trees can be built from the segments $s_{1}, \ldots, s_{n}$ by modifying the Bentley-Ottmann sweep for segment intersection [3, Chapter 7]: just remove the faster segment on any intersection. Since the total complexity of the trees is linear (every segment is removed at most once), the sweep completes in $O(n \log n)$ time. We remark that our algorithms do not need to construct the trees in the $x t$-plane exactly; we will store only the combinatorial structure of every tree (Section 2).

When a VIP is expected to arrive at the airport, the airspace has to be closed temporarily. All that is known about the VIP is the length $L$ of its ego, which measures for how long the approach will be closed. If the closure starts at time $T$, then all aircraft with entry times in the interval $(T, T+L)$ will be put into a holding pattern and will effectively enter the approach at time $T+L$. We assume that the sequence of aircraft does not change at the exit from holding (which is mostly true in the real world). Thus equivalently, the aircraft entrance is delayed until $T+L$, at which time they all enter in the same order in which they arrived (Fig. 1, right). Let $r_{i}(T, L)$ denote the time when aircraft $i$ will land; our cost function is the total sum the landing times

$$
D(T, L)=\sum_{i=1}^{n} r_{i}(T, L)
$$

Minimizing $D(T, L)$ is the same as minimizing the total delay $D(T, L)-\sum_{i=1}^{n} r_{i}$ caused by the VIP; therefore will call $D(T, L)$ also the delay.

To avoid trivialities we assume that there is a lower and an upper bound on the location of $T$ (otherwise, one would trivially set $T>t_{n}$ or $T<t_{1}-L$ ).

\subsection{Our Contributions}

We consider several problems:

Delay minimization (Section 2): Given $L$, find $T$ to minimize $D(T, L)$. To solve the problem in $O(n \log n)$ time we store the combinatorial structure of platoons in a forest which can be updated efficiently as the closure interval slides along the $t$ 
axis. The forest may be of independent interest, as it can be viewed as a juste milieu between storing platoons as lists and storing the full platoon trees in the $x t$-plane (the former does not have enough structure to allow for efficient updates, while the latter contains "too much" structure and therefore is hard to update).

Ego query (Section 3): The query version of delay minimization. We give a nearlyquadratic-time algorithm to compute the function $D(T, L)$ for all $L$ and $T$ (a simple example shows that the function can have quadratic complexity). We show that the marginal minimum $f(L)=\min _{T} D(T, L)$ of $D(T, L)$ has $O\left(n^{2} \alpha(n)\right)$ complexity and can also can be built in nearly-quadratic time. Knowing the function $f(L)$ allows one to give logarithmic-time answer to the delay minimization query "Given $L$, report $T$ that minimizes $D(T, L)$ ".

The Harmless President problem (Section 4): Given $T$, find maximum $L$ for which $D(T, L)$ is 0 . This is the dual problem to delay minimization: for the latter we assumed that the VIP would be willing to shift $T$ so as to minimize the delay - this could be an unrealistic assumption since VIPs have tight schedules; the solution to the Harmless President problem helps modest but busy VIPs who cannot reschedule the entry into the airspace but are willing to curb their egos so as to do no harm to the people (of course, the majority of politicians are such, so this is the most practical problem). The Harmless President problem can be solved in nearly-quadratic time using our results for the Ego query problem: after the function $D(T, L)$ is built we can find, for the given $T$, the largest $L$ such that $(T, L)$ is in the 0-level (complement of the support) of $D(T, L)$. We show that the 0-level actually has linear complexity and can be built in $O(n \log n)$ time; thus the Harmless President problem can be solved in $O(n \log n)$ time. In addition, using the 0-level, one can solve the query version of the Harmless President problem in logarithmic time per query.

Naturally, our algorithms are applicable to arbitrary scenarios of temporary traffic disruption: train track closure, farmers machinery (or geese, or kids) crossing a rural road, walkway blockage, etc. The algorithms can also be extended to handle (multiplelane) roads where passing is allowed. Some proofs and details are omitted from this version and will appear in the full paper.

\subsection{Related Work}

Traffic jam formation is a vast research area heated by enormous real-life importance of the field. Many studies-ranging from on-site experimental measurements to simulation and modelling to purely theoretical developments-have been performed over the years. Various traffic flow characteristics were explored, most notably the fundamental diagram of the flow (the relationship between flow rate, vehicle speed and density), aiding in understanding flow breakdown, jam formation and other processes occurring in the traffic. Not attempting to survey the huge amount of literature on the subject, we refer to books and surveys $[7,8,5]$. A related domain of active research is motion information gathering: traffic participants (be it cars, aircraft, ships, trains, pedestrians, birds or other animals) are being tracked using mobile phones, GPS navigators, specialpurpose devices, etc. One popular form of processing of the gathered data is information summarization, in particular - trajectory clustering; for some recent work see, e.g., [4] and references thereof. 


\section{Delay Minimization}

In this section we consider the following problem: Given $L$, find $T$ to minimize $D(T, L)$. First we discuss a simple $O\left(n^{2}\right)$ algorithm to solve the problem. For a more efficient solution we introduce a special data structure and assign colors to aircraft.

Our first goal is to calculate the landing times $r_{i}$ for all aircraft. Define the platoon structure $\mathcal{P}$ to be the list of aircraft platoons sorted by the entry times of head aircraft.

Lemma 1. The platoon structure $\mathcal{P}$ can be computed in $O(n \log n)$ time.

Proof. Construct the lists $T_{1}=\left(t_{1}, t_{2}, \ldots, t_{n}\right)$ and $T_{2}=\left(\tau_{i_{1}}, \tau_{i_{2}}, \ldots, \tau_{i_{n}}\right)$, sorted from lowest to highest value. For every pair of corresponding elements $t_{j}$ and $\tau_{j}$ store pointers to each other. Consider the maximum landing time $\tau_{i_{n}}$ and the corresponding entering time $t_{k}$ (where $i_{n}=k$ ). Aircraft $S_{k}=\left\{i_{k}, i_{k+1}, \ldots, i_{n}\right\}$ form a platoon with aircraft $i_{k}$ in the head. Put the platoon at the beginning of $\mathcal{P}$ and delete the aircraft from the lists $T_{1}$ and $T_{2}$. Repeat until $T_{1}$ and $T_{2}$ are empty. By construction, each element in $\mathcal{P}$ is a sorted list of aircraft of one platoon, and platoons in $\mathcal{P}$ are sorted by the entering times of their lead aircraft. The constructive part takes $O(n)$ time as each aircraft is deleted from $T_{1}$ and $T_{2}$ only once. The bottleneck is sorting $T_{2}$ which gives the total $O(n \log n)$ time for constructing the platoon structure.

Next note that there exists an optimal closure interval with the starting point $T$ equal to one of the entry times $t_{1}, \ldots, t_{n}$; otherwise we can slide the interval to the left along the time axis without changing its length until we reach some $t_{i}-$ this would not increase the delay. The simple solution is thus to consider all intervals $\left(t_{i}, t_{i}+L\right)$ separately, calculating the platoon structure for each interval in $O(n)$ time and comparing the resulting values of $D(T, L)$; this leads to an overall $O\left(n^{2}\right)$-time algorithm.

The above approach is not efficient, as it involves recomputing the delay $n$ times. For a more efficient algorithm we will slide the interval of length $L$ from left to right along the time axis, updating the delay $D(T, L)$ as a function of $T$. To facilitate the update we will introduce an upgraded version of the platoon structure, storing each platoon in a tree (Section 2.2).

\subsection{Aircraft Flying through Rainbows}

We introduce several colors to distinguish between how aircraft are affected by the closure. Let $t_{i}(T, L) \in\left\{t_{i}, T+L\right\}$ denote the time when aircraft $i$ enters the approach given that the airspace is closed during the interval $(T, T+L)$. Call aircraft $i$ delayed if it lands later than its scheduled landing time $r_{i}$, i.e., if $r_{i}(T, L)>r_{i}$; otherwise (i.e.,

\begin{tabular}{l|c|l|l|l}
\hline & $\begin{array}{l}\text { undelayed \& un- } \\
\text { affected }\end{array}$ & $\begin{array}{l}\text { undelayed \& dir. } \\
\text { affected }\end{array}$ & $\begin{array}{l}\text { delayed \& dir. } \\
\text { affected }\end{array}$ & $\begin{array}{l}\text { delayed \& ind. } \\
\text { affected }\end{array}$ \\
\hline platoon head & & impossible & \\
\hline following aircraft & &
\end{tabular}

Fig. 2: Overview of the colors, based on the properties of the aircraft before the closure and their interaction with the current closure interval. 
if $\left.r_{i}(T, L)=r_{i}\right)$ aircraft $i$ is undelayed. Say that an aircraft $j$ is directly affected by the closure interval $(T, T+L)$ if $t_{j} \in(T, T+L)$, i.e., if $t_{j}(T, L)=T+L>t_{j}$ (note that $j$ may still be undelayed, because it was following a slow aircraft even without the closure). Say that $i$ is indirectly affected if it is delayed but is not directly affected: $r_{i}(T, L)>r_{i}, t_{i}(T, L)=t_{i} \geq T+L$ (i.e., landing of $i$ is delayed because another aircraft in front of $i$ is directly affected).

We define seven aircraft colors (Fig. 2; see also Fig. 1, right):

dark green aircraft $i$ is an undelayed head of a platoon: $t_{i}(T, L)=t_{i}$ and $r_{i}(T, L)=$ $r_{i}=\tau_{i}$,

red aircraft $i$ is a delayed directly affected aircraft that would have been dark green (head of a platoon) if the airspace was not closed: $T<t_{i}<T+L, t_{i}(T, L)=$ $T+L$, and $r_{i}(T, L)>r_{i}=\tau_{i}$,

yellow aircraft $i$ is a delayed aircraft that is indirectly affected (through some red aircraft) and that originally, before introducing an airspace closure, was dark green: $T+L<t_{i}(T, L)=t_{i}, r_{i}(T, L)>r_{i}=\tau_{i}$,

light green aircraft $i$ is an undelayed aircraft that is not the head of a platoon: $t_{i}(T, L)=t_{i}$ and $r_{i}(T, L)=r_{i}>\tau_{i}$,

purple aircraft $i$ is a delayed aircraft that is directly affected and that originally, before introducing an airspace closure, was light green: $T<t_{i}<T+L, t_{i}(T, L)=T+L$, $r_{i}(T, L)>r_{i}>\tau_{i}$,

orange aircraft $i$ is a delayed aircraft that is indirectly affected (through some red aircraft) and that originally, before introducing an airspace closure, was light green: $T+L<t_{i}(T, L)=t_{i}, r_{i}(T, L)>r_{i}>\tau_{i}$,

blue aircraft $i$ is an undelayed aircraft that is directly affected: $T<t_{i}<T+L$, $t_{i}(T, L)=T+L, r_{i}(T, L)=r_{i}$; an aircraft can be blue only if originally, before the closure, it was light green.

The colors indicate whether an aircraft is delayed or not, and if originally, before introducing approach closure, the aircraft headed a platoon or not. A dark green aircraft (head of a platoon) can become red or yellow, but never blue, purple, light green or orange; similarly, a light green aircraft (not a head of a platoon), cannot become red or yellow (refer to Fig. 2, 3).

We will slide the closure interval $(T, T+L)$ to the right, starting from $T=t_{1}-L$, updating the colors of the aircraft and the delay function $D(T, L)$ for all $T=t_{i}(1 \leq i \leq n)$. Aircraft change their colors when:
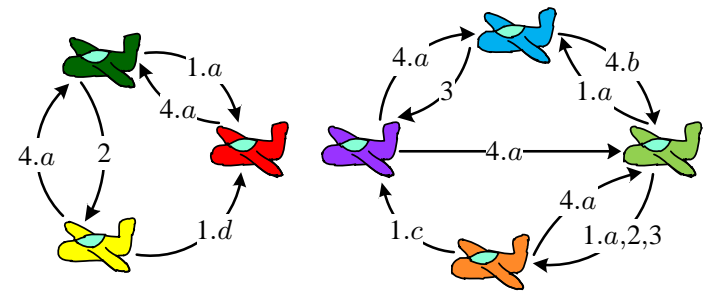

Fig. 3: Change of the aircraft colors

1. the right end of the interval hovers over the starting point of some aircraft $j$, i.e., when $t_{j}(T, L)$ changes from $t_{j}$ to $T+L$. If $j$ was (a) dark green, then it becomes red, and the rest of the aircraft in the platoon following $j$ become orange, (b) light green, then it becomes blue, (c) orange, then it becomes purple, (d) yellow, then it becomes red. 
2. two consecutive platoons $p_{j}$, with red head aircraft $j$, and $p_{k}$, with dark green head aircraft $k$, merge, i.e., $T+L+\tau_{j}-t_{j}>\tau_{k}$. This event changes the color of $k$ to yellow and the rest of the aircraft from platoon $p_{k}$ from light green to orange.

3. the interval pushes some aircraft out of a platoon. More precisely, when $j$ is a blue aircraft in a platoon $p_{k}$ with dark green head aircraft $k$, i.e., $t_{k}<T<t_{j}<T+L$, and $T+L+\tau_{j}-t_{j}=\tau_{k}$, then new platoon $p_{j}$ with head aircraft $j$ is separated from platoon $p_{k}$. This event changes the color of $j$ from blue to purple and the colors of the rest of the aircraft in the new platoon from light green to orange.

4. at every step the aircraft $i$ can change its color back to light or dark green. Its color right before this event can be red, purple, blue, light or dark green.

(a) if $i$ was red then it becomes dark green, if $i$ was purple then it becomes light green. There can be following changes in other aircrafts' colors: (i) if there were yellow aircraft, some of them can become dark green, (ii) if there were orange aircraft, they can become light green, (iii) if there were other purple aircraft, some of them can become blue.

(b) if $i$ was blue, then it becomes light green.

\subsection{Platoon Tree Structure}

We now introduce the platoon tree structure that will allow us to update $D(T, L)$ efficiently while sliding the closure interval. Instead of representing each platoon as a sorted list as before, now we store it in a tree whose nodes are sorted by increasing approach-entry times top-to-bottom and left-to-right among siblings, and are also sorted by desired speeds increasing top-to-bottom but decreasing left-to-right among siblings (Fig. 4). Specifically:

- the root of the tree is the head of the platoon,

- if node $j$ is a child of node $i$ then $t_{i}<t_{j}$ and $v_{i}<v_{j}$

- if $j$ is the left sibling of $i$ then $t_{i}>t_{j}$ but $v_{i}<v_{j}$. Moreover, the same inequalities hold for $i$ and any descendant $j^{\prime}$ of $j: t_{i}>t_{j^{\prime}}$ but $v_{i}<v_{j}^{\prime}$.

This tree structure has one nice property that we are going to use later on: consider a platoon $p$ consisting of several aircraft $\{j, j+1, \ldots, m\}$, and the corresponding tree structure $\mathcal{T}$. Let the closure interval $(T, T+L)$ for some $T=t_{i}$ contain the entry times $\left\{t_{i+1}, \ldots, t_{i+k}\right\}$ of $k$ aircraft from $p\left(t_{j}<t_{i+1}\right.$ and $\left.t_{i+k}<t_{m}\right)$. Mark the nodes in $\mathcal{T}$

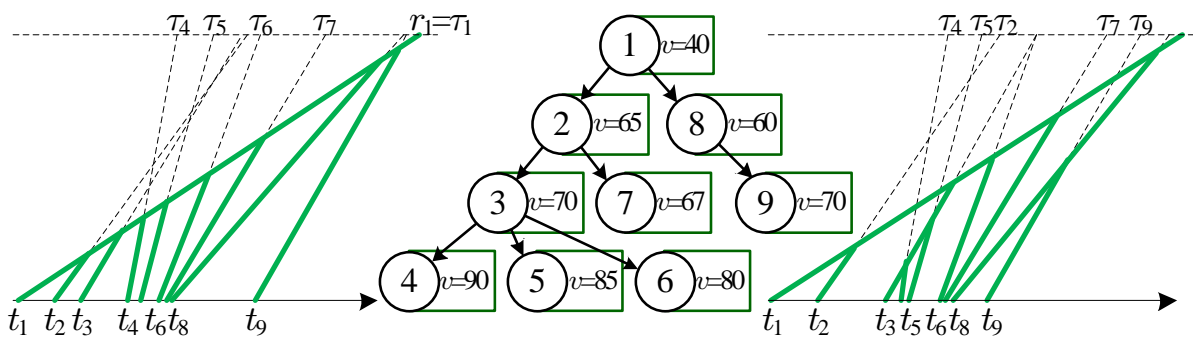

Fig. 4: Platoon tree for aircraft with desired velocities $(40,65,70,90,85,80,68,60,70)$ - listed in the order of increasing entry times. Note that the same platoon tree may correspond to different platoons on the $t x$-space (shown left and right) 
that correspond to the aircraft affected by the interval (see Fig. 5). Then the marked nodes form several subtrees, i.e., there is no unmarked node with a marked parent. The number of these subtrees is equal to the number of "sub-platoons" starting at point $T+L$, and the delay depends only on the sizes of the marked subtrees and the landing times of their root nodes.

A platoon tree can be constructed from a platoon list in linear time by scanning the list

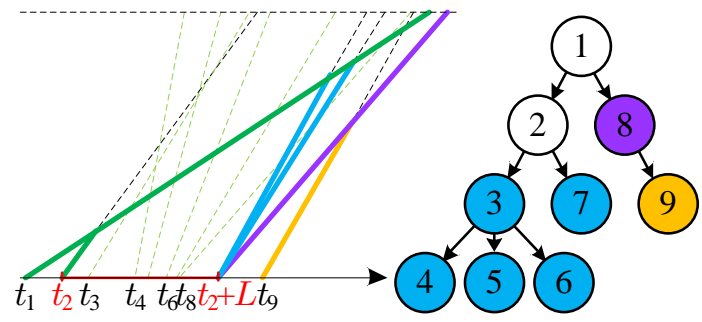

Fig. 5: The property of the platoon tree structure illustrated on the platoon from the left example in Fig. 4. Left: Trajectories in $t x$-space after the closure $\left(t_{2}, t_{2}+L\right)$. Right: Colored nodes of the tree are affected by the interval. They form three subtrees, and there are three "sub-platoons" entering the approach at the same time $t_{2}+L$

in the increasing order of $t_{i}$ (i.e., starting from the head) and making node $i+1$ the child of $i$ if $v_{i+1}>v_{i}$. Otherwise, i.e., if $v_{i+1}<v_{i}$, we go up the tree to the first node $j$ on the $i$-to-root path that has $v_{j}<v_{i+1}$ and make $i+1$ the rightmost child of $j$. Any node is visited only once, as the construction is equivalent to traversing the tree in the depth-first-search order. Therefore, the total time it takes to construct a platoon tree structure is $O(n)$.

\subsection{Rainbow Algorithm}

In this section we present an algorithm to find an optimal interval of given length $L$ that minimizes the total delay introduced in $O(n \log n)$ time.

Let $\mathcal{F}(T)$ be a forest structure consisting of platoon trees that contain all aircraft with starting times greater than $T$. All the aircraft with a starting point before $T$ cannot be delayed, therefore we do not keep track of them in $\mathcal{F}(T)$. The trees are sorted by the starting times of their head aircraft. All the nodes in a tree have the same landing time as the root of the tree (the head of the platoon). If the root is delayed, all the nodes in the corresponding tree are delayed by the same amount. Therefore, to calculate the total delay we need to know the number of nodes in the tree, and the difference between the delayed arrival time and the scheduled arrival time. In every node $i$ we store $t_{i}, \tau_{i}$ and $r_{i}$. Moreover, we store the current values of $t_{i}(T, L)$ and $r_{i}(T, L)$ in the root nodes of the trees in $\mathcal{F}(T)$ that are affected by the closure interval. We also color the nodes of the trees in $\mathcal{F}(T)$ into the corresponding colors of the aircraft (see Fig. 6).

If the closure $(T, T+L)$ falls in between two platoons, then $\mathcal{F}(T)$ consists of several trees with red roots, possibly followed by several trees with yellow roots, and several trees with dark green roots in the end (Fig. 6a). The red roots correspond to the heads of the platoons that are directly affected by the closure, their starting time is $T+L$. All the following aircraft in these platoons are orange, they have the same landing times as their roots. The yellow roots correspond to the heads of the platoons that are indirectly affected by the closure. Their landing time is the same as the landing time of the last red head of a platoon. 


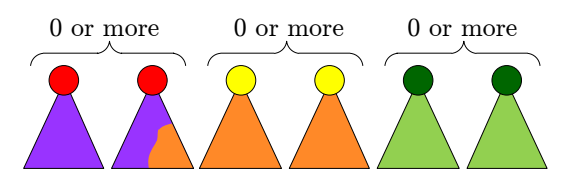

(a) The closure interval falls in between of two platoons

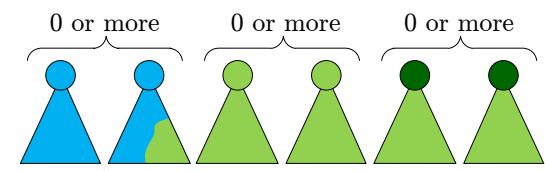

(b) Zero-delay airspace closure

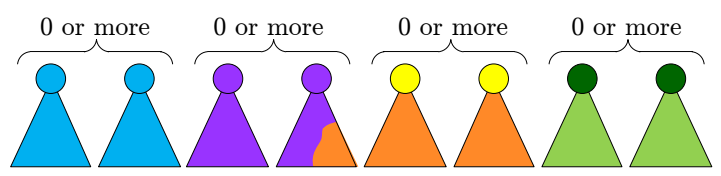

(c) General case of $\mathcal{F}(T)$

Fig. 6: $\mathcal{F}(T)$ consists of trees with blue, purple, red, light green, and dark green roots

If the closure interval falls in the middle of a platoon, then $\mathcal{F}(T)$ can consist of several trees with blue roots, followed by several trees with light green roots (Fig. 6b), or of several trees with blue roots, followed by trees with purple roots, then with red roots, yellow roots, and dark green roots in the end (Fig. 6c). The first case corresponds to a zero-delay airspace closure. There are several aircraft with starting times moved to $T+L$, but their landing times are not affected by the closure. In this case, we can stop the algorithm and report a zero-delay position of the closure. In further description of the algorithm we assume that this case does not occur. In the second case, the trees with the purple, red and yellow roots contain aircraft that are delayed.

At every step of the algorithm, we slide the closure interval from $\left(t_{i}, t_{i}+L\right)$ to $\left(t_{i+1}, t_{i+1}+L\right)$, and remove the leftmost root $i$ from $\mathcal{F}(T)$. The children of $i$ move one level up and become roots of their subtrees. After that we update the colors of some nodes and the value of $D(T, L)$. Node $i$, before the removal from $\mathcal{F}(T)$, can be blue, light green, purple, red or dark green. It cannot be yellow or orange, as all aircraft of these colors must follow a delayed aircraft, which has to be in $\mathcal{F}(T)$ before such nodes.

After sliding the interval and removing $i$ from $\mathcal{F}(T)$

- some yellow and dark green nodes can become red (if the right end of the closure interval slides over the starting times of these aircraft). However red nodes cannot become yellow, they only change their color back to dark green once they are removed from $\mathcal{F}(T)$;

- some dark green nodes can become yellow, or some yellow nodes can become dark green;

- some blue roots in $\mathcal{F}(T)$ can become purple (if $i$ was blue and the right end of the interval "pushes" some blue sub-platoons out of the current platoon), or some purple roots can become blue (if $i$ was purple and some of the following aircraft fall back under the current platoon).

We omit the proof of the following lemma (refer to the full version of this paper for the details):

Lemma 2. After sliding the closure interval from $\left(t_{i}, t_{i}+L\right)$ to $\left(t_{i+1}, t_{i+1}+L\right)$, forest $\mathcal{F}(T)$ and delay $D(T, L)$ can be updated in $O(\log n)$ time. 


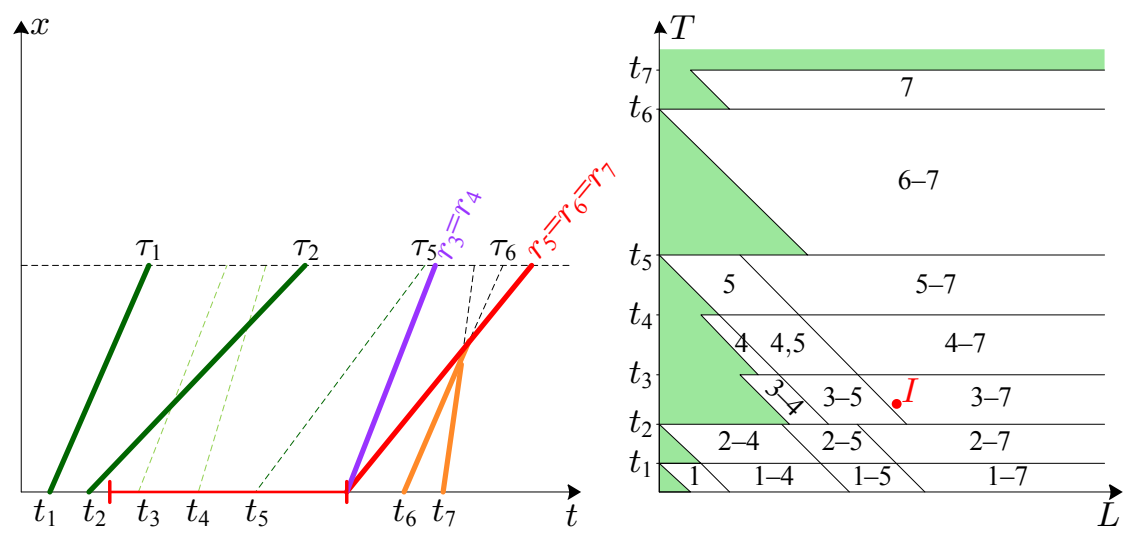

Fig. 7: The diagram (right) for the instance on the left. (Green is the 0-impact region; see Section 4.) The aircraft influenced by the closure are indicated in each cell. Point $I$ corresponds to the closure interval from the left

The rainbow algorithm performs $n$ iterations, each in $O(\log n)$ time. Therefore, we get:

Theorem 1. The rainbow algorithm finds an optimal interval of given length that minimizes the delay in $O(n \log n)$ time.

\section{Ego Query}

We now consider the query version of delay minimization, i.e., answering queries of the type "Given the length $L$ of the ego, report the best closure start moment $T$ ". We build an $O\left(n^{2} \alpha(n)\right)$-complexity function to answer such queries in $O(\log n)$ time using $O\left(n^{2} \log n\right)$ preprocessing time.

Extending the idea from the previous section, we start from building the delay diagram (Fig. 7) - the subdivision the $L T$-space into cells such that the delay $D(T, L)$ is given by the same function within a cell. We build the diagram separately in each strip between the lines $T=t_{i}$ and $T=t_{i+1}$; clearly, the function $D(T, L)$ stays combinatorially the same for all $T \in\left(t_{i-1}, t_{i}\right)$ (however not all lines $T=t_{i}$ are necessarily edges of the diagram, so we may actually compute a slightly finer subdivision than needed). We fix an arbitrary point $T \in\left(t_{i-1}, t_{i}\right)$ and increase $L$ watching for events when

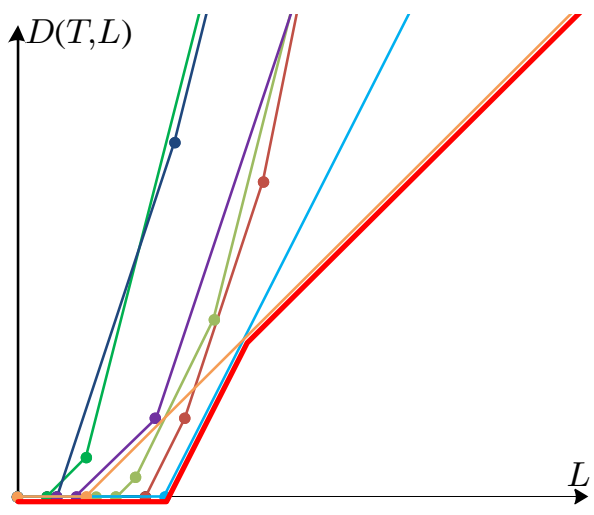

Fig. 8: The lower envelope (red) for the instance from Fig. 7. Every color corresponds to a horizontal line $T=t_{i}$ in the diagram (the functions for diagonal edges of the diagram are not shown because the minimum is attained on the horizontal edges) 
$D(T, L)$ changes; the events and updates are analogous to those considered in the previous section (note that in the previous section we kept the length $L$ fixed an varied the starting time $T$, but we can modify the algorithm to work for fixed $T$ and varied $L$; we omit the details from this version). We spend $O(n \log n)$ time per strip, constructing the diagram in overall $O\left(n^{2} \log n\right)$ time (note that the diagram may have quadratic complexity overall, e.g., if the trajectories of aircraft pairwise do not cross, i.e., if each aircraft is a platoon by itself).

Since $D(T, L)$ changes linearly within each cell of the diagram, for any $L$, the optimal $T$ will lie on an edge of the diagram. We graph $D(T, L)$ as functions of $L$ for all diagram edges on a single plot (Fig. 8); since the diagram has $O\left(n^{2}\right)$ complexity, there are $O\left(n^{2}\right)$ segments on the plot, and the optimal $T$ as a function of $L$ is the lower envelope of the segments. The function has $O\left(n^{2} \alpha(n)\right)$ complexity and can be constructed from the segments in $O\left(n^{2} \log n\right)$ time [6].

Theorem 2. We can build a data structure of $O\left(n^{2} \alpha(n)\right)$ size in $O\left(n^{2} \log n\right)$ time that can answer ego queries in $O(\log n)$ time.

\section{Algorithms for Harmless VIPs}

The 0 level of the function $D(T, L)$ corresponds to closure intervals $(T, T+L)$ that do not introduce any delay into the traffic. Obviously, the $T$-axis belongs to the 0 level $(D(T, L)=0$ for $L=0$ ) and for every $T$ there is a maximum $L$ for which $D(T, L)=$ 0 . Thus, the right boundary of the level is a $T$-monotone curve, and the level is (the right half of) a tree with the $T$-axis as the trunk. Moreover, since the 0 level is a union of cells of the delay diagram and edges of the diagram are straight-line segments, the right boundary of the level is a polygonal curve. We therefore refer to the 0 level as the Christmas tree (Fig. 9; see also Fig. 7).

Clearly, the Christmas tree can be constructed in $O\left(n^{2}\right)$ time using results from the previous section. In this section we show how to compute the tree in linear time (after platoons in the absence of the closure have been identified, which can be done in
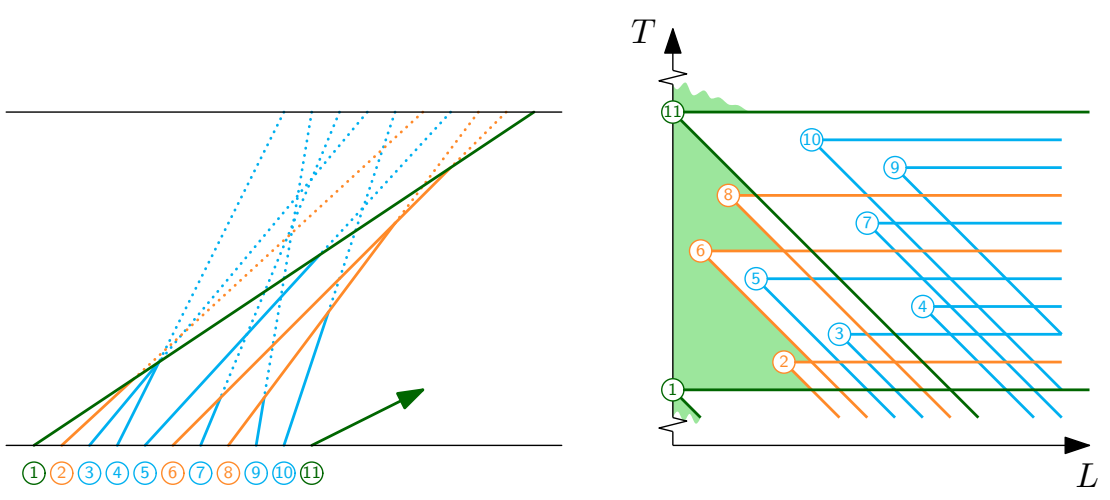

Fig. 9: Left: A platoon with 10 aircraft; $i=1, h=11$. Right: the corresponding sub-Christmastree. Platoon heads are green, and other aircraft that influence the shape of the tree are orange; the other aircraft are blue. Circles are the points $\left(\tau_{j}-\tau_{i}, t_{j}\right)$ 
$O(n \log n)$ time. The crucial observation is that the Christmas tree can be built platoonby-platoon: if the closure interval $(T, T+L)$ contains the entry time $t_{i}$ for a platoon head $i$, then $i$ is delayed and $(L, T)$ is not in the Christmas tree; thus, it is enough to consider only intervals lying between entry times of two consecutive heads. Moreover, the right boundary of the Christmas tree must touch the $T$-axis at $t_{i}$ (since for $T$ infinitesimally smaller than $t_{i}$ we have $D(T, L)>0$ for infinitesimally small $L$ ). Hence, entry times of platoon heads split the Christmas tree into "sub-Christmas-trees" touching the $T$-axis at their bottoms and tops (Fig. 9, right). In what follows we will focus on building the (sub-)tree for one platoon, headed by $i$, and assume that the closure starts between $t_{i}$ and $t_{h}$ where $h$ is the head of the platoon that follows $i$ 's platoon (so the aircraft in $i$ 's platoon are $i, \ldots, h-1$ ). That is, our goal will be to build the (sub-)tree in the strip between the lines $T=t_{i}$ and $T=t_{h}$.

Let $j \in\{i, \ldots, h\}$ be an aircraft from $i$ 's platoon or $h$. Aircraft $j$ is delayed iff it is directly affected $\left(t_{j} \in(T, T+L)\right)$ and lands later than $i$ does $\left(T+L+\tau_{j}-t_{j}>\tau_{i}\right)$, i.e., iff $(L, T)$ lies in the wedge between the rays $T=t_{j}$ and $T+L=t_{j}+\tau_{i}-\tau_{j}$ emanating from the point $\left(\tau_{j}-\tau_{i}, t_{j}\right)$ (refer to Fig. 9, right). Our (sub-)tree is the complement of the union of the wedges for all aircraft $i, \ldots, h$. To build the tree observe that it is the Pareto envelope (set of undominated points) of the apexes of the wedges in a sheared copy of the $L T$-space. Since aircraft are sorted along the $T$ axis, the envelope can be build in linear time: scan the apexes from top to bottom and for each apex test whether it lies to the left or to the right of the diagonal line through the previous apex; in the former case include it in the envelope, in the latter case throw it away.

Theorem 3. We can build a data structure in $O(n \log n)$ time that can answer a query “Given $T$, report the largest $L$ for which $D(T, L)=0$ " in $O(\log n)$ time.

\section{Acknowledgments}

We thank the anonymous reviewers for their helpful comments. Irina Kostitsyna is supported in part by the Netherlands Organisation for Scientific Research (NWO) under project no. 612.001.106. Maarten Löffer is supported by the Netherlands Organisation for Scientific Research (NWO) under grant no. 639.021.123. Valentin Polishchuk's work was supported by the Academy of Finland grant 1138520.

\section{References}

1. T. Allard, 2012. Personal communication.

2. ATCOs, 2013. Personal communication.

3. M. d. Berg, O. Cheong, M. v. Kreveld, and M. Overmars. Computational Geometry: Algorithms and Applications. Springer-Verlag, Santa Clara, CA, USA, 3rd ed. edition, 2008.

4. K. Buchin, M. Buchin, J. Gudmundsson, M. Löffler, and J. Luo. Detecting commuting patterns by clustering subtrajectories. Int. J. Comput. Geometry Appl., 21(3):253-282, 2011.

5. F. L. Hall. Traffic stream characteristics. Traffic Flow Theory. US Federal Highway Administration, 1996.

6. J. Hershberger. Finding the upper envelope of $\mathrm{n}$ line segments in $\mathrm{O}(\mathrm{n} \log \mathrm{n})$ time. Inf. Process. Lett., 33(4):169-174, 1989.

7. A. May. Traffic Flow Fundamentals. Prentice Hall, 1990.

8. W. McShane, R. Roess, and E. Prassas. Traffic Engineering. Prentice Hall, 1998. 